\title{
Unorthodox Presentation of Colorectal Cancer With Multiple Fistulas and Multiple Abscesses
}

\author{
Hernan Gonzalez Monroiga, d, Fermin Lopez Rivera ${ }^{\mathrm{a}}$, Jessica Castellanos Diaz ${ }^{\mathrm{a}}$, \\ Joham Senior Marino ${ }^{\mathrm{b}}$, Ricardo Rivera Hernandez ${ }^{\mathrm{c}}$
}

\begin{abstract}
Colorectal cancer is the third most lethal cancer in the United States and is the third most common type of cancer. In 2016, 134,490 cases of colorectal cancer were diagnosed in the United States. The diagnosis is usually made with screening colonoscopy on asymptomatic patients, while other patients are diagnosed after presenting symptoms such as hematochezia, iron deficiency anemia, changes in bowel movements, and abdominal pain. Also on emergency surgical procedures, a tumor may be found. We present a case of a 50-year-old Hispanic woman, G3P3AO, with a past medical history of hypertension, major depressive disorder and hysterectomy in 2003 without any toxic habits or family history of colon cancer or inflammatory bowel disease. Patient arrived with left lower quadrant pain, fever, and loss of appetite and was treated with IV antibiotics for an acute diverticulitis. Patient had multiple visits to urgency room for fecal material that discharged from the vaginal area and multiple intraabdominal abscesses in addition to obstruction. On imaging studies, patient had rectovaginal fistula and multiple abscesses. The patient's clinical condition became more complicated with enterocutaneous fistula and enterocolonic fistula 4 months later, suggesting inflammatory bowel disease. Sigmoid adenocarcinoma was diagnosed on the second colonoscopy. The clinical presentations of this patient with multiple abscesses and fistulas are commonly found on inflammatory bowel disease and can also be found in complicated intraabdominal infections. Rectovaginal fistulas which were the first fistula more commonly are caused by obstetric complications. Other common causes associated include surgery, inflammatory bowel disease, radiation therapy, and malignancy. These findings can mask the diagnosis of colorectal cancer and make the diagnosis challenging.
\end{abstract}

Keywords: Colorectal adenocarcinoma; Crohn's disease; Rectovaginal fistula; Enterocutaneous fistula; Acute diverticulitis

Manuscript submitted November 7, 2017, accepted December 12, 2017

${ }^{a}$ Internal Medicine Department, San Juan City Hospital, San Juan, Puerto Rico ${ }^{\mathrm{b}}$ Gastroenterology Department, University District Hospital, San Juan, Puerto Rico

'Radiology Department, University of Puerto Rico, San Juan, Puerto Rico ${ }^{\mathrm{d} C o r r e s p o n d i n g ~ A u t h o r: ~ H e r n a n ~ G o n z a l e z ~ M o n r o i g, ~ I n t e r n a l ~ M e d i c i n e ~ D e-~}$ partment, San Juan City Hospital, 151 Calle Cesar Gonzalez Apartamento 3301, San Juan 00918 Puerto Rico. Email: hgonzalez@sanjuanbautista.edu

doi: https://doi.org/10.14740/jmc2959w

\section{Introduction}

At present, colorectal cancer is the third most lethal cancer in the United States and is the third most common type of cancer [1]. The incidence differs from developed and undeveloped countries [2]; 134,490 cases of colorectal cancer were diagnosed in the United States on 2016. Patients can be symptomatic or asymptomatic, depending on the location or progression and may present with different clinical features. The diagnosis is usually made with screening colonoscopy on asymptomatic patients, while other patients are diagnosed after presenting symptoms such as hematochezia, iron deficiency anemia, changes in bowel movements, and abdominal pain. Also on emergency surgical procedures, tumor may be found $[3,4]$. Uncommon presentations of colon cancer can mimic diverticulitis, and malignant fistulas on rectosigmoid locations and also can be seen as multiple abscesses on different areas of the gastrointestinal system $[5,6]$.

\section{Case Report}

The case was a 50-year-old woman, G3P3AO, with a past medical history of hypertension, major depressive disorder and hysterectomy in 2003. The patient does not have any toxic habits or family history of colorectal cancer or inflammatory bowel disease; the only history of malignancy is her mother who was diagnosed with a brain tumor at the age of 71. Patient's first visit to emergency room (ER) was in August 2016 with symptoms that included suprapubic pain, flank pain and fever. The patient was managed with oral antibiotics for urinary tract infection and discharged home from ER. Three months later, the patient presented with similar symptoms, but this time, an abdomen-pelvis CT scan was remarkable for acute diverticulitis and was treated accordingly. Patient was discharged home after completing antibiotics and tolerating oral diet. Patient returned to ER on January 2017 with an acute abdomen, loss of appetite, fever, and nausea, and a CT scan with IV and PO contrast was repeated. The CT scan showed concentric thickening of the bowel wall with associated mural abscess, and fistulous connection between the sigmoid colon and vaginal cuff with rectal material on the vaginal cuff. Also a fistulous connection between the sigmoid colon and the left abdominal muscle was found. These findings were consistent 


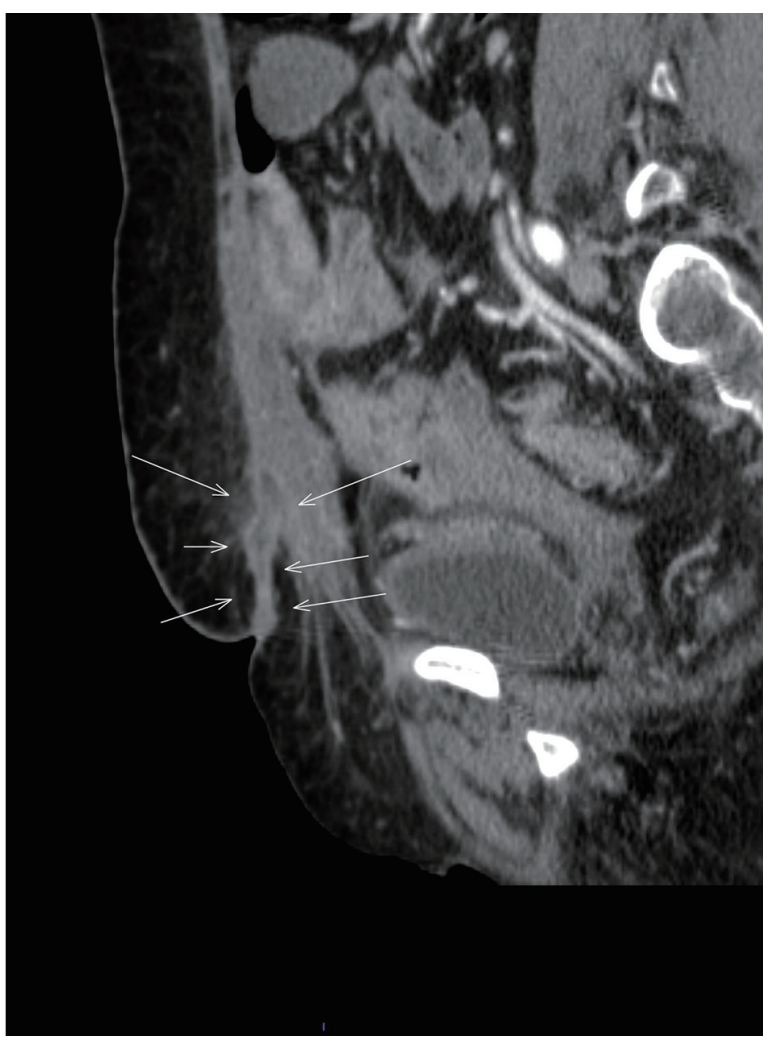

Figure 1. Abdominopelvic CT scan of sagittal view with IV and PO contrast (white arrows) showing fistulous tract and enterocutaneous fistula.

with colitis and multiple fistulas; however, colonoscopy was recommended to rule out malignancy. Colonoscopy was performed 2 months later after receiving IV antibiotics and biopsy

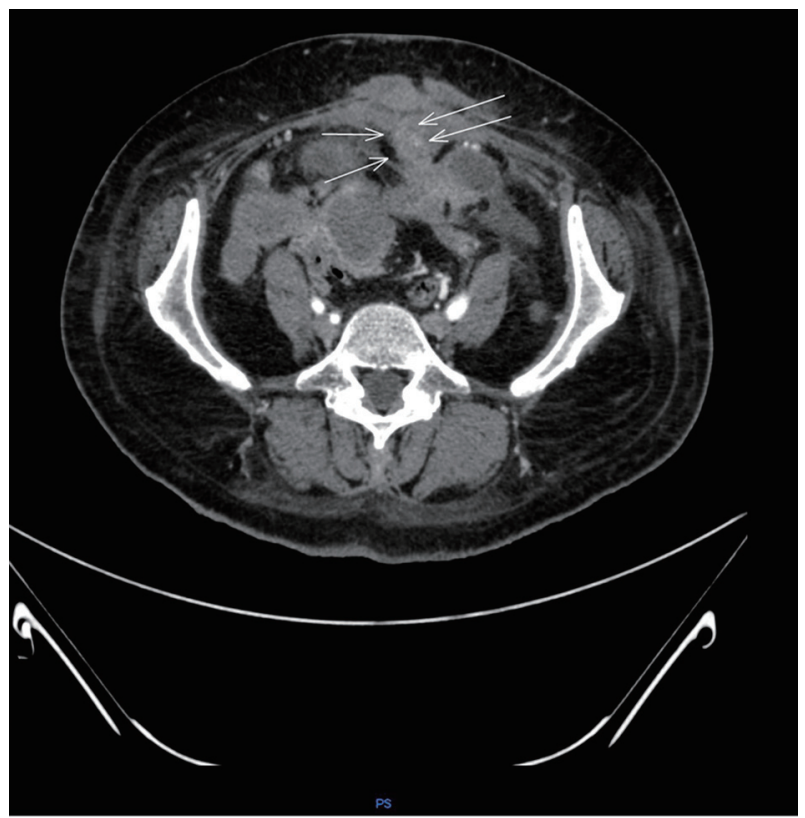

Figure 2. Abdominopelvic CT scan with IV and PO contrast transverse view (white arrows) pointing fistulous tract and enterocutaneous fistula.

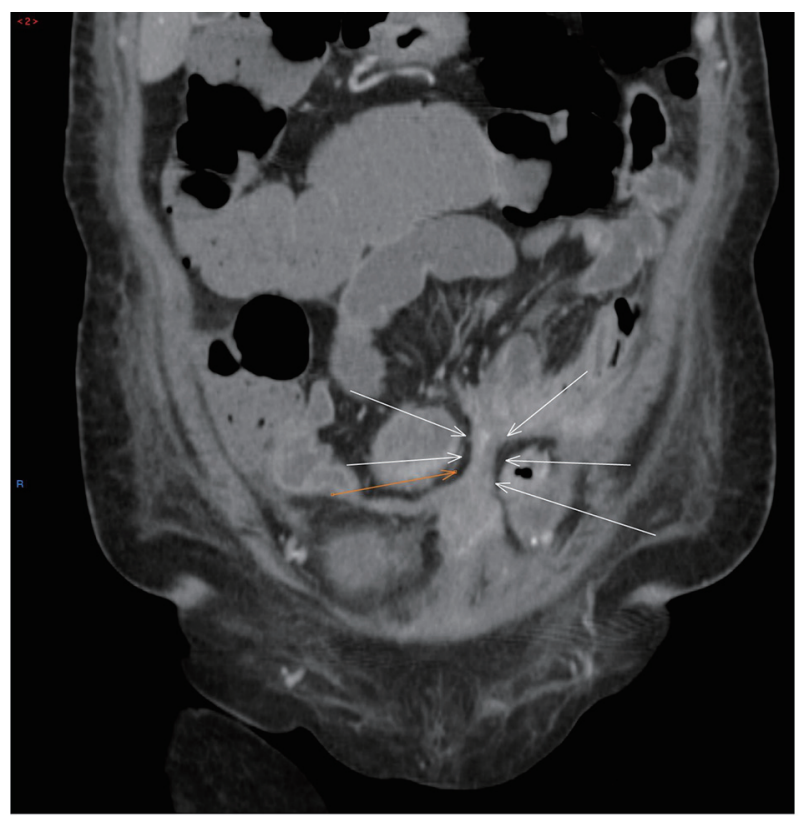

Figure 3. Abdominopelvic CT scan with $\mathrm{PO}$ and IV contrast coronal view showing colonic fistulous tract (white and orange arrows).

taken from the sigmoid colon was negative for malignancy. Patient's symptoms worsened with active purulent secretions from abdominal wall and secretions of vaginal area that she described as fecal material. Patient was seen by gynecology service for evaluation of rectovaginal fistula, abundant fecal material was found at the vagina, and patient was referred to colorectal surgeon. Patient symptoms recurred by May 2017;

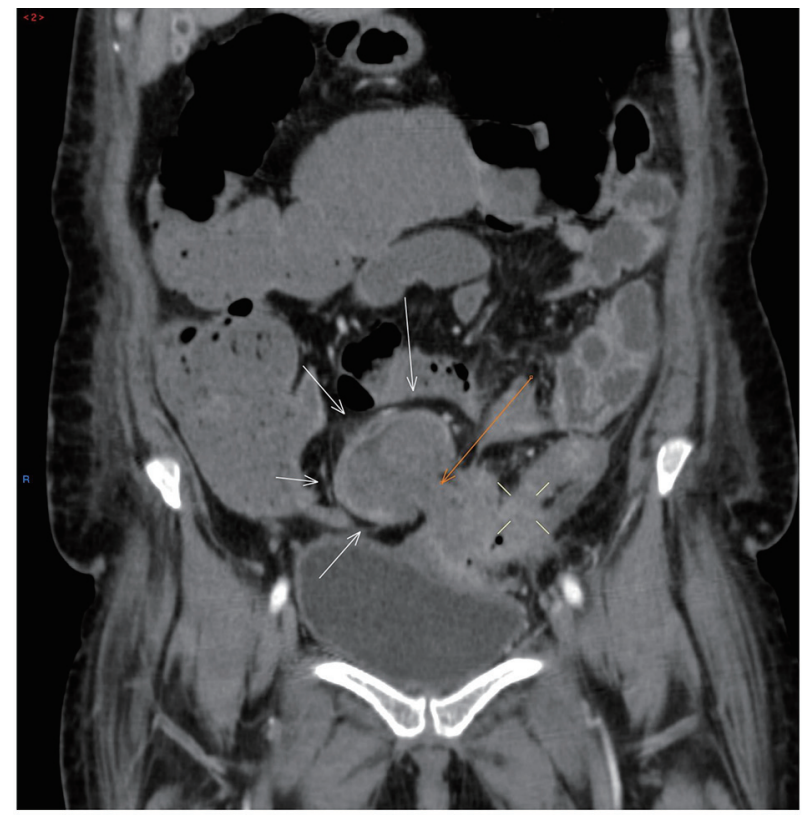

Figure 4. Abdominopelvic CT scan with PO and IV contrast coronal view showing large intraabdominal abscess (white arrows, orange arrow) showing bowel wall (four white lines showing another intraabdominal abscess). 


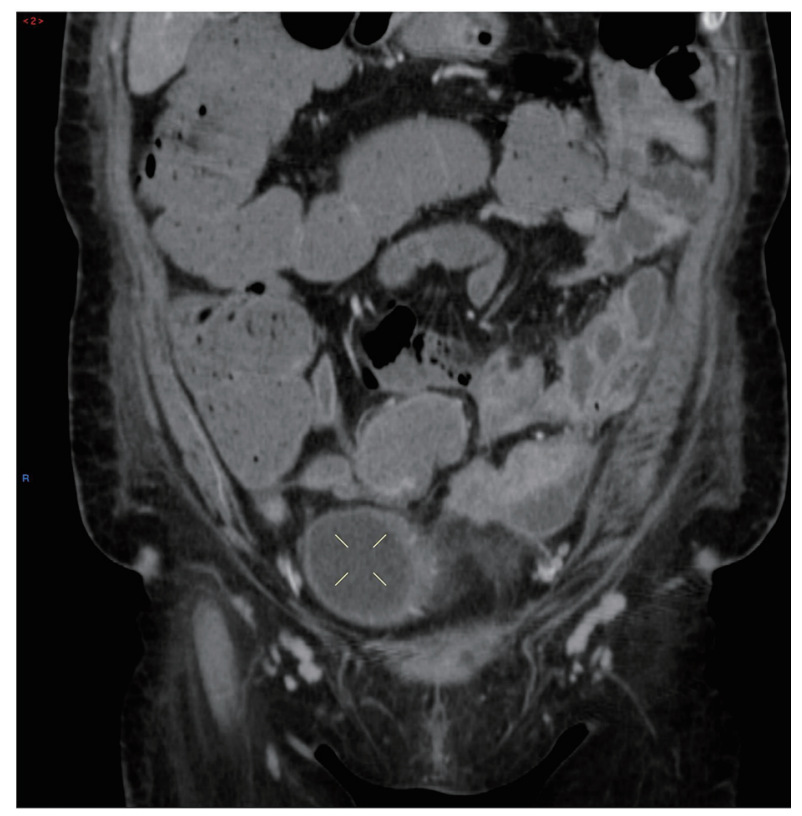

Figure 5. Coronal abdominopelvic CT scan with IV and PO contrast (four white lines) showing large intraabdominal abscess.

however, this time, the patient developed new enterocolonic, enterocutaneous, and colovaginal fistula seen on abdomen-pelvis CT scan with wall thickening and stranding in mesentery.

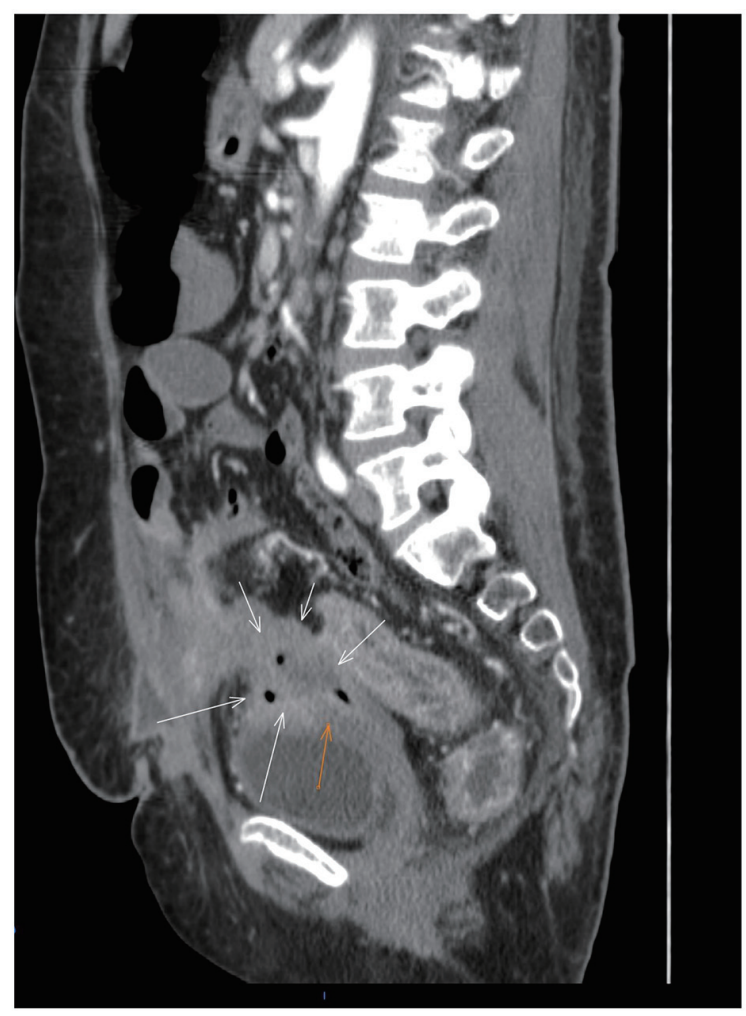

Figure 6. Abdominopelvic CT scan with IV and PO contrast sagittal view (white arrows) showing fistulizing tract and (orange arrow) showing rectovaginal fistula.

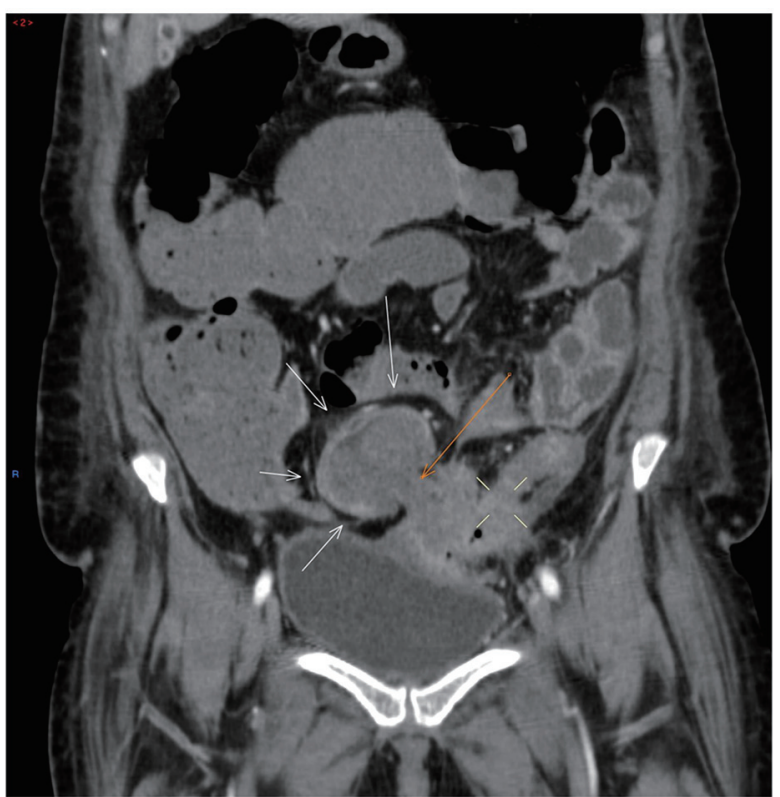

Figure 7. Abdominopelvic CT scan with PO and IV contrast coronal view showing colonic fistulous tract (white and orange arrows).

At this moment, Crohn's disease was suspected and was transferred for inflammatory bowel disease specialist evaluation. Colonoscopy was repeated and a biopsy from a mass on the sigmoid colon was taken and pathology reported an adenocarcinoma of the sigmoid colon (Figs. 1-7).

\section{Discussion}

A fistula can be defined as an abnormal connection between two body structures that are not supposed to be connected. The different fistulas can be classified in accordance to the different anatomic connections. External gastrointestinal fistulas result from connection between the gastrointestinal tract and the skin and drains to the outside part of the body and are called enterocutaneous. Internal fistulas do not drain through the skin and are connections between the gastrointestinal tract and adjacent organs. The origin and insertion is used for the nomenclature of each fistula [7].

Trauma associated with childbirth is the most common cause of rectovaginal fistula which was the first fistula that appeared in this patient. Tears of the perineum that extend to the bowel or procedures to facilitate delivery on prolonged and difficult labor can produce this type of fistula. Crohn's disease is the second most common cause of inflammatory process on the gastrointestinal tract associated with the development of fistula and also abscesses are common complications of this disease. Malignancy and radiation therapy on the pelvic area can also predispose patients to develop fistulas. Finally, surgical procedures are other common causes $[8,9]$.

The development of multiple abscesses and fistulas on adjacent parts to the colon is a common complication of Crohn's disease and can also be associated with complicated infectious process along the gastrointestinal tract. This patient had a hys- 
terectomy which is a known risk factor for the development of fistula; however, the hysterectomy was done 14 years before the appearance of the fistulas, suggesting that the surgical procedure was not the primary cause of the development of fistula and abscesses. Prolonged labor is also unlikely to be the primary cause of the development of multiple fistulas because patient time frame of last pregnancy exceeds more than 15 years and this presentation would have occurred earlier. Patient also had a diverticulitis that could result in the complications seen in this case such as multiple abscesses and rarely development of fistula. The patient persisted several months with multiple abscesses that probably led to the development of new fistula seen later. However, the rectovaginal fistula was present at the moment when multiple abscesses were found on the imaging studies. Crohn's disease is another possible pathology associated with fistula and multiple abscesses were ruled out on two colonoscopies where tissue biopsy was taken.

In this case, the colorectal cancer of the sigmoid was diagnosed on the second colonoscopy. It is atypical due to patient age, negative family history, and initial presentation with rectovaginal fistula and multiple abscesses. From this case, we can learn that colorectal cancer can mimic other conditions such as Crohn's disease or complicated infectious process and colon adenocarcinoma should be part of the differential diagnosis on patients presenting with fistulas and multiple abscesses. The symptoms, laboratory findings, and clinical presentation can be very similar because the inflammation is the pathophysiology process of origin that can result from infectious process, autoimmune disease or malignancy. Leukocytosis, elevated inflammatory markers are common laboratory findings seen in the three different pathologies. Symptoms such as abdominal pain, loss of appetite, weight loss, fever, and changes in bowel movement can be seen in patients with either infectious process, inflammatory bowel disease or malignancy. This case shows that colorectal cancer can present with atypical symptoms and workup should continue aggressively until a final diagnosis is made.

Inflammatory bowel disease can be related with extraintestinal manifestations that are not found in colorectal cancer. For example aphthous ulcers, ankylosing spondylitis, eye manifestations including uveitis and scleritis are not typically found on colorectal cancer. Some skin manifestations that can be found include pyoderma gangrenosum, erythema nodosum, and cutaneous vasculitis. Pyoderma gangrenosum is not expected to be found in patients with colorectal cancer and is most commonly associated with inflammatory bowel disease [10]. However, it has been associated with hematologic malignancies such as IGA monoclonal gammopathy, leukemia, lymphoma and myeloma and on rheumatic disorders such as ankylosing spondylitis and rheumatoid arthritis [11-13]. These clinical findings are not found on all patients with inflammatory bowel disease; however, they can help with establishing the correct diagnosis. In the case of this patient, none of these extraintestinal manifestations were found. Anti-Saccharomyces cerevisiae antibodies (ASCA) can be found in patients with Crohn's disease in comparison to colorectal cancer. Imaging studies in some cases can have apple core sign that is most commonly associated with colorectal cancer; however, in some cases, Crohn's disease can also be found. Skip lesions and cobblestone ap- pearance are found in colonoscopy on patients with Crohn's disease. Colonoscopy findings in colorectal cancer can be variable depending on the stage of the tumor and size can limit the evaluation of the entire colon if the mass is causing complete obstruction. On biopsy histologic findings can be different in the case of Crohn's disease. Non-caseating granulomas may be found with transmural lymphocytic infiltration. Colorectal adenocarcinoma histology can be variable depending on the stage and the degree of differentiation. The degree on gland formation is variable, and abundant glands are associated with highly differentiated and moderate differentiated tumors, which can be surrounded by tall columnar cells, and extensive necrosis with debris. Non-caseating granulomas are not found on colorectal cancer. These are differences in colorectal cancer and Crohn's disease; however, Crohn's disease increases the risk of colorectal cancer, so patients with Crohn's disease that develop colorectal cancer can have characteristics of both conditions in colonoscopy and histology. Screening colonoscopy is different in patients with Crohn's disease from normal population without history of colorectal cancer and is done $8-10$ years after diagnosis every 1 - 2 years.

\section{Acknowledgments}

The authors wish to acknowledge the assistance of Alan Santiago M.D. attending of the Internal Medicine program of San Juan City Hospital, in the diagnosis, guidance of management and the preparation of the manuscript of this article.

\section{Financial Support}

No source of financial support to disclose.

\section{Conflict of Interest}

We have no conflict of interest to declare.

\section{References}

1. Siegel RL, Miller KD, Jemal A. Cancer statistics, 2016. CA Cancer J Clin. 2016;66(1):7-30.

2. Jemal A, Bray F, Center MM, Ferlay J, Ward E, Forman D. Global cancer statistics. CA Cancer J Clin. 2011;61(2):69-90.

3. Moreno CC, Mittal PK, Sullivan PS, Rutherford R, Staley CA, Cardona K, Hawk NN, et al. Colorectal cancer initial diagnosis: screening colonoscopy, diagnostic colonoscopy, or emergent surgery, and tumor stage and size at initial presentation. Clin Colorectal Cancer. 2016;15(1):67-73.

4. Moiel D, Thompson J. Early detection of colon cancerthe kaiser permanente northwest 30-year history: how do we measure success? Is it the test, the number of tests, the stage, or the percentage of screen-detected patients? Perm J. 2011;15(4):30-38. 
5. Tsai HL, Hsieh JS, Yu FJ, Wu DC, Chen FM, Huang CJ, Huang YS, et al. Perforated colonic cancer presenting as intra-abdominal abscess. Int $\mathrm{J}$ Colorectal Dis. 2007;22(1):15-19.

6. Alvarez JA, Baldonedo RF, Bear IG, Alvarez P, Jorge JL. Anaerobic liver abscesses as initial presentation of silent colonic cancer. HPB (Oxford). 2004;6(1):41-42.

7. Berry SM, Fischer JE. Classification and pathophysiology of enterocutaneous fistulas. Surg Clin North Am. 1996;76(5):1009-1018.

8. Andreani SM, Dang HH, Grondona P, Khan AZ, Edwards DP. Rectovaginal fistula in Crohn's disease. Dis Colon Rectum. 2007;50(12):2215-2222.

9. Saclarides TJ. Rectovaginal fistula. Surg Clin North Am. 2002;82(6):1261-1272.

10. Farhi D, Cosnes J, Zizi N, Chosidow O, Seksik P,
Beaugerie L, Aractingi S, et al. Significance of erythema nodosum and pyoderma gangrenosum in inflammatory bowel diseases: a cohort study of 2402 patients. Medicine (Baltimore). 2008;87(5):281-293.

11. Bennett ML, Jackson JM, Jorizzo JL, Fleischer AB, Jr., White WL, Callen JP. Pyoderma gangrenosum. A comparison of typical and atypical forms with an emphasis on time to remission. Case review of 86 patients from 2 institutions. Medicine (Baltimore). 2000;79(1):37-46.

12. Binus AM, Qureshi AA, Li VW, Winterfield LS. Pyoderma gangrenosum: a retrospective review of patient characteristics, comorbidities and therapy in 103 patients. $\mathrm{Br}$ J Dermatol. 2011;165(6):1244-1250.

13. Ahronowitz I, Harp J, Shinkai K. Etiology and management of pyoderma gangrenosum: a comprehensive review. Am J Clin Dermatol. 2012;13(3):191-211. 